\title{
Legislación ambiental y minera en Cuba. Aciertos y desaciertos
}

Environmental and mining legislation in Cuba. Successes and failures

\author{
C. Yaelsy, Lafita Cobas ${ }^{1}$ \\ (iD) https://orcid.org/0000-0001-6453-6128 \\ Universidad de Oriente, Cuba
}

\section{Como referenciar este artículo:}

\author{
Lafita., C., Y. (2021). Legislación ambiental y minera en Cuba. Aciertos y desaciertos. Revista \\ Ciencia Jurídica y Política, 146-164.
}

\section{RESUMEN}

La actividad minera provoca daños considerables al medio ambiente por ello es preciso, que cada país adopte las normas legales que contribuyan a la mitigación de estos. Con este propósito Cuba, ha aplicado una política sustentada por una base jurídica, administrativa y organizativa que permita su instrumentación, así como la realización de una gestión integral e integrada del ambiente. Por lo que es preciso la existencia de una estructura organizativa estatal, dotada de autoridad y competencia suficiente, para la aplicación, ejecución y control de la política y la gestión ambiental encaminadas al logro de un desarrollo económico y social sostenible. En tal sentido se traza como objetivo de esta investigación realizar un análisis de las normas jurídicas existentes en Cuba que permiten la protección del medio ambiente ante los embates de la minería. Para la realización de esta investigación se utilizaron los métodos teóricos de las Ciencias Jurídicas entre los que se encuentran: Análisis histórico-jurídico y Análisis exegético-jurídico, así como parte de la metodología propia de las Ciencias Sociales se utilizó la técnica de investigación de revisión de documentos.

Palabras Claves: medio ambiente, minería, legislación.

\begin{abstract}
The mining activity causes considerable damage to the environment, so it is necessary that each control adopt the legal norms that contribute to the mitigation of these. For this purpose, Cuba has implemented a policy supported by a legal basis, administrative and organizational basis that allows for its implementation, as well as the implementation of a comprehensive and integrated management of the environment. Therefore, the existence of a state organizational structure is necessary, with sufficient authority and competence for the implementation, execution and legal policy and environmental management aimed at achieving sustainable economic and social development. In this sense, the objective of this research is to carry out an analysis of the legal norms existing in Cuba that allow the environmental protection in the face of mining. For the realization of this research, theoretical methods of the legal sciences were used, among which are: Historical- legal analysis and exegetic analysislegal, as well as the own methodology of the Social Sciences was used the technique of research review of documents.
\end{abstract}

Key Word: Environment, mining, legislation
Recibido: 07.01.21
Aceptado: 17.03.21

\footnotetext{
${ }^{1}$ Doctora en Ciencias Jurídicas, Profesora Titular de la Universidad de Oriente, Directora de Capital Humano Empresa Geominera Oriente, Santiago de Cuba, Cuba, email: laguajiradelyunque@gmail.com, yaelsy.lafita@scgeomin.minem.cu.
} 


\section{1.- INTRODUCCIÓN}

La actividad minera es una de las actividades que más genera contaminación y afecta al medio ambiente, por tanto, para minimizar y mitigar los impactos negativos que esta actividad provoca, en muchas partes del mundo se han adoptado de forma prioritaria el concepto de Desarrollo Sostenible, de manera que ha sido asumido recientemente en la Constitución de la República de Cuba al establecer que el Estado tiene dentro de sus fines esenciales promover un desarrollo sostenible que asegure la prosperidad individual y colectiva, y obtener mayores niveles de equidad y justicia social, así mismo fue definido en la Ley No. 81 de Medio Ambiente de Cuba (1997) como el:

Proceso de elevación sostenida y equitativa de la calidad de vida de las personas, mediante el cual se procura el crecimiento económico y el mejoramiento social, en una combinación armónica con la protección del medio ambiente, de modo que se satisfacen las necesidades de las actuales generaciones, sin poner en riesgo la satisfacción de las necesidades de las generaciones futuras.

Como todo sistema social, el Desarrollo Sostenible tiene sus propios principios”. Entre estos, aplicables a la actividad minera, se pueden citar los siguientes: (1) "respetar y cuidar la comunidad de los seres vivientes"; (2) "mejorar la calidad de vida humana"; (3) "conservar la vitalidad y diversidad de la Tierra"; (4) "reducir al mínimo el agotamiento de los recursos no renovables" y (5) "modificar las actitudes y prácticas personales referentes al medio ambiente" (Unión Mundial para la Naturaleza [UICN, por sus siglas en inglés], Programa de las naciones unidas para el medio ambiente [PNUMA] y Fondo mundial para la naturaleza [WWF, por sus siglas en inglés], 1991).

Cuba no ha estado exenta a esta problemática, ya que desde el siglo pasado existió un auge de la actividad minera, muestra de ello fue la extensión en 1902 de la mina El Cobre y el inicio de la explotación de hierro y manganeso en la región oriental, y posteriormente la explotación del yacimiento de Matahambre en Pinar del Río desde 1913 hasta 1997. En la década de los cuarenta se inicia la explotación de laterita para la 
extracción de níquel más cobalto y otros minerales como plata, tungsteno, oro y en los últimos tiempos la explotación de petróleo y gas.

La Constitución de la República de Cuba en su artículo 11 expresa que el Estado ejerce soberanía y jurisdicción: sobre el medio ambiente y los recursos naturales del país, en igual sentido promueve la protección y conservación del medio ambiente y el enfrentamiento al cambio climático, que amenaza la sobrevivencia de la especie humana, sobre la base del reconocimiento de responsabilidades comunes, pero diferenciadas; en igual sentido establece un orden económico internacional justo y equitativo y la erradicación de los patrones irracionales de producción y consumo; en igual sentido regula, que son de propiedad socialista de todo el pueblo, los yacimientos minerales, las minas, y los recursos naturales tanto vivos como no vivos dentro de la zona económica exclusiva de la República y le corresponde al Estado el dominio inalienable e imprescriptible del subsuelo, las minas y todos los recursos minerales.

De forma semejante, la Ley No. 76, Ley de Minas de fecha 21 de diciembre de 1994, declara en el artículo 4 la forma de propiedad de los recursos naturales y en el artículo 1 de la mencionada Ley se dispone como uno de sus objetivos la Política Minera de la nación, aprobada por acuerdo del Consejo de Estado de la República de Cuba en cumplimiento de lo dispuesto en el Capítulo III, Artículo 5 de esta norma jurídica.

Como podemos apreciar, en Cuba se ha venido prestando especial atención a la protección del medio ambiente en estrecha vinculación con el desarrollo económico y social del país, lo cual se materializó en la promulgación primeramente de la Ley No. 33 "De Protección del Medio Ambiente y el Uso Racional de los Recursos Minerales (1981) y posteriormente en la Ley No. 81, Del Medio Ambiente de 1997, que actualizó los principios, objetivos y conceptos básicos de la política ambiental cubana y los instrumentos para su materialización de acuerdo al desarrollo económico y social actual.

Nuestra Ley de Minas, aunque anterior a la ya citada Ley de Medio Ambiente, tuteló la preservación del medio ambiente en las actividades mineras, lo que 
complementó el Decreto No. 222, Reglamento de la Ley de Minas del 16 de septiembre de 1997.

Por todo lo anterior la presente comunicación persigue como objetivo realizar un análisis de las normas jurídicas existentes en Cuba que permiten la protección del medio ambiente ante los embates de la minería, de manera que se logre identificar las fortalezas y debilidades de estas y se puedan promover políticas proactivas y crear sinergias que coadyuven al desarrollo de la minería en nuestro país, con criterios de seguridad, sustentabilidad medioambiental, y socialmente aceptables.

\section{2.- MÈTODOS}

Para la realización de esta investigación se utilizaron los métodos teóricos de las Ciencias Jurídicas entre los que se encuentran:

Análisis histórico-jurídico, en atención al estudio de la evolución del ordenamiento jurídico en la protección al medio ambiente vinculada a la actividad minera en Cuba, para lograr una correcta comprensión de esta actividad y su ordenación en la actualidad. Análisis exegético-jurídico, para la realización de un examen de todos los textos legales contenidos en la investigación, y en específico las regulaciones vigentes en Cuba que contienen aspectos sobre el régimen jurídico que regula la protección al medio ambiente y su relación con la actividad minera.

Y como parte de la metodología propia de las Ciencias Sociales se utilizó la técnica de investigación de revisión de documentos, que consistió en la revisión bibliográfica de artículos científicos, legislaciones nacionales, informes y artículos de contenido afines con la investigación, y publicaciones periódicas, para conocer el estado de la ciencia, las tendencias nacionales e internacionales concernientes al tema de investigación.

Estos métodos han sido validados por expertos en Metodología de la Investigación Jurídica entre los que se encuentran Villabella Armengol (2012) , Carlos Manuel; Rodríguez Gómez, Gregorio et al.(2002), Pavo Rolando (2009); Jiménez Serrano, Pablo (1998) (2015),

Gómez Tapia (2018), quienes ha expresado: los métodos para investigación teórica o 
métodos teóricos se utilizan para recolectar datos y obtener informaciones necesarias, los mismos permiten operar a un nivel del pensamiento abstracto con conocimientos que se han condensado como son teorías, leyes, paradigmas, entre otros. Através de ellos se construye el discurso científico mediante el cual se argumentan y demuestran los nuevos conocimientos.

Los métodos teóricos generalmente reconocidos por los autores, y que son aplicables a la investigación jurídica, son los siguientes:1) Histórico-lógico.2) Análisis-síntesis.3) Abstracción-concreción.4) Inductivo-deductivo.5) Sistémico-estructural-funcional.6) Modelación y de la investigación empírica, el método de análisis de contenidos que se emplea fundamentalmente para hacer valoraciones cualitativas de documentos.

\section{3.- MEDIO AMBIENTE, MINERÍA Y DESARROLLO SOSTENIBLE}

Existen dos formas para desarrollar la minería, subterránea y a cielo abierto. La minería a cielo abierto es menos costosa y más productiva que la minería subterránea. La construcción de caminos mineros es de bajo costo y se produce en un tiempo razonablemente corto, lo que minimiza los costos finales de extracción. Facilita las labores de mantenimiento de equipos y la introducción de nuevas tecnologías. Esta minería es más agresiva pues ocasiona importantes afectaciones ambientales y sociales.

La toma de conciencia de los problemas derivados de la utilización inadecuada de los recursos naturales se manifiesta en la evolución del pensamiento económico al analizarse la relación hombre-medio ambiente. Antes del siglo XIX los paradigmas imperantes en Europa fueron el mercantilismo y el movimiento fisiocrático.

La doctrina mercantilista de los siglos XVI al XVIII, defendió la acumulación de recursos naturales no renovables como principal exponente de la riqueza de una nación. Los fisiócratas, consideraban a la tierra como principal fuente de riqueza; el objetivo económico se centraba en aumentar la producción y el rendimiento agrícola. No existía ninguna preocupación por la naturaleza. Se creía que la tierra constituía una fuente inagotable de recursos al servicio del hombre. 
Los economistas clásicos, escuela iniciada con Adam Smith, David Ricardo y que culmina con John Stuart Mill, defendían la propiedad privada, los mercados y la competencia como instrumentos para alcanzar el bienestar social, desconfiando de la intervención del gobierno en la regulación de la actividad económica. Bajo esta teoría, el trabajo pasó a ser considerado la principal fuente de riqueza. David Ricardo introduce el concepto de rendimientos decrecientes, según el cual, al aumentar los factores capital y trabajo, disminuyen los rendimientos. Desde un punto de vista microeconómico la cuestión ambiental se ha centrado en las denominadas "externalidades", surgidas de los planteamientos originarios de John Stuart Mill, quien predijo que el crecimiento económico continuado tendría efectos negativos para el medio natural (Riera, 1992).

Uno de los principales inconvenientes de la teoría clásica es que no toma en consideración las interdependencias entre el sistema natural y económico y la necesidad de un desarrollo equilibrado del medio ambiente. La oposición a la escuela clásica vino de la mano de las teorías económicas enunciadas a finales del siglo XIX por Carlos Marx y Federico Engels, los cuales consideraron que el progreso es el resultado de la explotación incontrolada de la naturaleza y deberá llevar al fracaso del capitalismo.

Según Carlos Marx, el punto de partida para el análisis de la crisis ambiental contemporánea está en la propia producción mercantil. Mientras la producción precapitalista de valores de uso tiene su límite en la satisfacción de las necesidades, la producción mercantil, para incrementar la ganancia, no tiene límite alguno. Esta diferencia tiene sus bases en el agotamiento de los recursos naturales a un ritmo nunca sospechado en la historia de la humanidad y en la generación ilimitada de desechos (Marx,1973).

La conservación de la vida en la Tierra y la gestión de los recursos naturales bajo un punto de vista global se ha convertido en un reto inaplazable para la perpetuación de la propia especie humana, sin embargo, esto no impide la extinción de especies, la pérdida de hábitat, la fragmentación de los ecosistemas, la contaminación de cuencas hidrográficas y en general la pérdida de la diversidad biológica (Vilamajó et al., 2002).

Vilamajó et al. (2002) afirman: la conservación de la diversidad biológica es hoy reconocida como uno de los problemas más apremiantes de la humanidad, relacionado 
estrechamente con el necesario desarrollo económico y social de las naciones", considerando además que, "es por la aparente contradicción entre conservación y desarrollo que los pueblos se preguntan cuándo y cómo pueden utilizar sus recursos sin que disminuya la capacidad de renovarse a sí mismo", a lo que podemos responder "sólo a partir de estrategias cuyos objetivos se centren en lograr el Desarrollo Sostenible.

Es preciso recordar desarrollo sostenible busca la integración que multidimensional, es decir que requiere el diseño e implementación de políticas públicas multisectoriales: económicas, sociales, culturales, educativas, de salud, ambientales, etc. Sin embargo, como en este artículo se hace mayor énfasis en la dimensión ambiental, cabe señalar que política ambiental es un conjunto de intervenciones que tienen el objetivo de reducir la degradación ambiental y/o resarcir los daños ya causados a través de mecanismos centralizados (impuestos, subsidios y permisos por emisiones, o establecimiento de estándares tecnológicos, de calidad ambiental y de emisiones) y descentralizados (derechos de propiedad, negociaciones y persuasión moral) (Lavín, 2015).

El desarrollo sostenible ha evolucionado de un concepto de justicia intergeneracional a uno de integración multidimensional, que requiere la implementación de una buena gobernanza para lograr el diseño de políticas públicas vinculantes y coherentes que creen sinergias intersectoriales y multidimensionales (Manzanares Garmendia 2020).

“Ante la degradación natural como antrópica de los ecosistemas, la restauración y rehabilitación ecológica, parecieran ser la última opción para la sobrevivencia de la especie humana y de la vida en general en el planeta" (Barrera et al., 2010). Para poderla llevar a cabo el hombre debería cambiar de actuar, darle el verdadero valor a la vida y entender que cada vez son menos las opciones para las generaciones futuras. Por ello es preciso que cada país adopte las normativas jurídicas necesarias para prevenir y mitigar el negativo impacto que sobre el medio ambiente tiene la actividad. 


\section{4.- DISCUSIÓN.}

Cada país instrumenta la política ambiental a aplicar a través de una base jurídica, administrativa y organizativa que la sustenta, por ello han ido adecuando la estructura y organización de su aparato a sus características y condiciones particulares.

Cuba no es la excepción y desde la promulgación de la Constitución de la República de Cuba el 24 de febrero de 1976, introdujo el tema ambiental, de manera legal, en el contexto cubano, lo que fuera ratificado en nuestra recién aprobada Carta Magna, en consonancia con lo que Ley No. 81 sobre Medio Ambiente estableció: Es necesario consagrar, como un derecho elemental de la sociedad y los ciudadanos, el derecho a un medio ambiente sano y a disfrutar de una vida saludable y productiva en armonía con la naturaleza, en tanto los seres humanos constituyen el objetivo esencial del desarrollo sostenible.

Análogamente lo anterior se complementó inicialmente con la Ley 1323 de Organización de la Administración Central del Estado, de 30 de noviembre de 1976, la que dispuso que el Comité Estatal de Ciencia y Técnica sería el encargado del establecimiento, la dirección y el control del Sistema Nacional de Protección del medio ambiente y el uso de los Recursos naturales, del mismo modo por acuerdo del Consejo de Ministros de igual fecha, se crea la Comisión Nacional de protección del Medio Ambiente y Conservación de los Recursos Naturales cuyas funciones fueron aprobadas el 24 de octubre de 1979, por ese mismo órgano, de manera que constituye el primer antecedente en materia de institucionalización de la esfera ambiental en nuestro país.

De igual manera las funciones y atribuciones otorgadas al Comité Estatal de Ciencia y Técnica, fueron transferidas mediante el Decreto Ley No. 147, de 21 de abril de 1994, De la Organización de la Administración Central del Estado al Ministerio de Ciencia, Tecnología y Medio Ambiente, creado por la propia norma jurídica.

No es hasta la promulgación de la Ley No. 81, Ley del medio ambiente, en la que se estableció en su título segundo el marco institucional del medio ambiente cubano. Esta legislación dispone las atribuciones que corresponden tanto a los organismos de la administración central del estado como a los órganos locales del Poder Popular, buscando la 
integración de los principios básicos, que deben regir la concepción de un sistema institucional ambiental que responda a las necesidades del país. Además, quedaron delimitadas las atribuciones y funciones del Ministerio de Ciencia, Tecnología y Medio Ambiente como rector en materia ambiental, así como se determinan los ámbitos de acción de todos los organismos de la administración central del estado respecto a la gestión y protección ambiental, tanto en esferas específicas de protección como en elementos del ambiente construido.

Acorde con la Política ambiental que había venido experimentando el país, se crearon otras instituciones que han contribuido al perfeccionamiento de la gestión del Ministerio de Ciencia, Tecnología y Medio entre las que se destacan: los Centros de Gestión Ambiental, de Inspección y Control Ambiental, de información, Divulgación y Educación Ambiental, y áreas Protegidas.

De igual manera la protección al medio ambiente en la actividad minera en Cuba ha estado dirigida a todos los sectores de la economía cubana, entre ellos el sector estatal o público, el sector cooperativo, el sector mixto y el sector privado. Este último cobra auge a partir del año 2010, momento en que se promulga la Resolución No. 32 del Ministerio de Trabajo y Seguridad Social, conllevando a un relanzamiento de este sector en la economía cubana ya casi desaparecido desde el triunfo revolucionario en enero de 1959. Mas tarde fue ratificado la existencia de este sector privado mediante diversas normas jurídicas del propio ministerio, tales como la Resolución No. 42 fecha 22 de agosto del 2013, las resoluciones 11 y 12 de fecha 29 de junio de 2018, y la Resolución No. 104 de 22 de octubre de 2019, vigente actualmente, de manera que en ellas se autorizaron las actividades que guardan relación con la actividad minera y que pueden ejercer las personas naturales, entre las que encontramos: Productor vendedor o recolector vendedor de materiales con fines constructivos. Integra las actividades de Cantero, Granitero, Productor o recolector vendedores de artículos de alfarería u otros materiales con fines constructivos y Elaborador vendedor de artículos de mármol.

Por consiguiente, en la mayoría de estas actividades se exige el cumplimiento de las regulaciones de los ministerios de Ciencia, Tecnología y Medio Ambiente en cuanto a los 
niveles de contaminación ambiental, así mismo el cumplimiento de las regulaciones establecidas para la extracción, explotación y procesamiento de los recursos minerales, sin embargo, a pesar de estar regulado, en la práctica no siempre se cumple.

Evidencia de lo anterior se palpa a través de las autorizaciones emitidas por la Oficina Nacional de Recursos Minerales, rectora de la actividad minera en Cuba, no existiendo autorización para personas privadas para el ejercicio de estas actividades en la minería sin embargo son desarrolladas sin tener en cuenta las obligaciones que generan una vez que exploten el medio ambiente. Igualmente fue corroborado por criterios emitidos por funcionarios de esta institución quienes refirieron que no existe un control exhaustivo de las personas naturales que realizan estas actividades vinculadas a la minería. De igual manera dentro de los organismos rectores que regulan estas actividades, no figura el Ministerio de Energía y Minas sino el Ministerio de la Construcción y la Agricultura fundamentalmente, lo que dificulta en la práctica el control de estas actividades.

Mención aparte merece el Programa Nacional de Medio Ambiente y Desarrollo cubano, donde se establece la protección de los recursos mineros, disponiendo como objetivos a alcanzar: la protección y manejo adecuado de los recursos minerales, de manera que se logre un desarrollo sostenible de la actividad minera; mantener actualizado el alcance anual de las reservas minerales del país; apoyar el trabajo de evaluación de las áreas que deben ser declarados cotos mineros y áreas mineras reservadas con el fin de compatibilizar la minería con el entorno donde se desarrolla y fortalecer el trabajo de rehabilitación en los casos que procedan.

La Oficina Nacional de Recursos Minerales, subordinada al Ministerio de Energía y Minas y el Ministerio de Ciencia, Tecnología y Medio Ambiente constituyen los organismos encargados de la supervisión y control de la ejecución y funcionamiento del marco regulatorio minero ambiental en Cuba, dispuesto de esta manera por la Ley No. 76, ley de Minas ya mencionada anteriormente y su Reglamento.

La primera calificada como Autoridad Minera, tiene entre sus funciones, según lo establece la mencionada Ley de Minas, "la del control de la ejecución de los planes de preservación del control ambiental y de las medidas para mitigar el impacto 
ambiental", con lo que se expresa la voluntad de minimizar los daños producidos por la actividad minera generadora de grandes impactos al medio ambiente y a la vez promover un mecanismo preventivo al respecto.

Partiendo de uno de los principios enunciados en la referida Ley de Minas, que expresa que "la ejecución de la actividad minera tiene en cuenta la competencia que la legislación le confiere al Ministerio de Ciencia, Tecnología y Medio Ambiente en asuntos ambientales", el artículo 10 del referido Reglamento de la Ley de Minas, obliga a la Autoridad Minera a compatibilizar previo al otorgamiento de los derechos mineros todas las solicitudes presentadas con el referido Ministerio de Ciencia, Tecnología y Medio Ambiente, para evitar lesionar los intereses ambientales en dichas áreas. Se refuerza dicha compatibilización, al exigirse en el trámite de presentación de las solicitudes para realizar actividades de explotación y procesamiento la aprobación de microlocalización para la inversión, que conlleva a su vez un trámite de conciliación con diversos organismos, entre ellos, la Autoridad Ambiental.

Es preciso destacar que el proceso de compatibilización entre los organismos que intervienen es responsabilidad estatal y se realiza antes de la aprobación de la concesión, por ello la gestión ambiental minera comienza desde el momento en que se inicia las operaciones autorizadas.

La ley de minas establece diversas obligaciones a los concesionarios que los conducen a la preservación del medio ambiente, la minimización del impacto ambiental y la correspondiente rehabilitación, entre las que encontramos: preservar adecuadamente el medio ambiente y las condiciones -ecológicas del área objeto de la concesión, elaborando estudios de impacto ambiental y planes para prevenir, mitigar, controlar, rehabilitar y compensar dicho impacto derivado de sus actividades; tanto en dicha área como en las áreas y ecosistemas vinculados a aquéllos que puedan ser afectados.

Lo anterior conlleva a que los concesionarios tramiten y obtengan la licencia ambiental previo al inicio de los trabajos y la realización de estudios de impacto ambiental para los concesionarios de explotación y procesamiento enunciado en los 
artículos 37, del Reglamento de la Ley de Minas y 120, de la referida Ley de Medio Ambiente.

Otras de las obligaciones establecidas en la ya mencionada ley de minas es preservar la salud y vida de los trabajadores aplicando las normas de seguridad e higiene del trabajo establecidas en las disposiciones vigentes; lo que ha materializado a través de la protección que se provee al hombre como factor fundamental en el ejercicio de las actividades mineras y para ello fue aprobado la Resolución No. 158 del Ministerio de Energía y Minas, que establece el Reglamento de Seguridad Minera (2014), el que persigue como objetivo establecer los requisitos generales para la seguridad y salud de los trabajadores; los diferentes métodos de minería: subterránea, a cielo abierto (incluidas las salinas) y marina, así como prevenir los daños que la actividad minera puede ocasionar a la salud humana, al medio ambiente y al patrimonio, mediante la identificación de los riesgos inherentes a la misma y su adecuada gestión y aprovechar los recursos minerales de modo racional y en un ambiente seguro.

De igual manera es responsabilidad del concesionario controlar, en las etapas de explotación y procesamiento y en la pequeña minería, la efectividad del procesamiento de tratamiento de los residuales; lo que garantiza el uso eficiente de los recursos naturales utilizados y que se dé el destino final a los desechos generados en los procesos productivos que no afecte al medio ambiente, enunciado en la citada Ley No. 81 .

Así mismo, la Ley de Minas instituye que las minas pueden ser cerradas de manera temporal o definitiva por daños al medio ambiente. Especial interés reviste el cierre de la mina, pues el concesionario está en la obligación de establecer las medidas de restauración y rehabilitación del entorno y llevar a cabo el programa de restauración de la superficie afectada y suministrar un informe sobre las afectaciones provocadas al medio ambiente, lo que debe hacerse en correspondencia con las condiciones ambientales exigidas en la licencia ambiental y que no es privativo de las áreas explotadas si no que es extensivo a las áreas de investigación que no son de interés para continuar los trabajos.

Para obtener los fondos necesarios para la mitigación de los impactos directos e indirectos de la actividad minera y la rehabilitación de las áreas, el concesionario está 
obligado a crear una reserva para los gastos derivados de la protección del medio ambiente y que la fija en un límite mínimo del 5\% del total de la inversión, lo que fuera previsto en el Reglamento de la Ley de Minas en su artículo 87.

Para lograr la protección al medio ambiente no solo se deja a la voluntad de los concesionarios y a las obligaciones que por ley le vienen atribuidas, sino que se establecen otras medidas administrativas, determinando cuales son las contravenciones que pueden ser cometidas por los concesionarios, de igual manera se establecen las medidas que se adoptan en cada caso entre las que se encuentran las multas y las medidas accesorias que correspondan (Ley de Minas, 1994, arts. 87 y 97).

Consecuentemente entre las obligaciones de los concesionarios se encuentran:

a) Ejecutar algunas de las fases de la actividad minera sin el debido título minero,

b) Explorar un recurso mineral, distinto de los que autoricen en la concesión,

c) Alterar las informaciones estadísticas y técnicas solicitadas por la autoridad minera,

d) Oponerse a la realización de la inspección estatal,

e) No llevar los registros que reflejen adecuadamente el desarrollo de la actividad minera,

f) Alterar los datos de los registros que reflejan adecuadamente el desarrollo de la actividad minera,

g) No demarcar el área de la concesión minera,

h) Alterar la demarcación de la concesión minera.

Como se puede apreciar en materia de responsabilidad ambiental, entre las contravenciones previstas en el Reglamento de la Ley de Minas el Decreto Ley No. 222 (1997), no aparece ninguna que directa y expresamente se refiera a la tutela del medio ambiente, sin embargo se establece en el artículo 97 de esta propia norma, la posibilidad de reprimir el incumplimiento de los trabajos a que están obligados los concesionarios según sus respectivos títulos, por ende su alcance se extiende en algunos casos a las obligaciones ambientales. 
Por su parte el incumplimiento reiterado de las medidas establecidas para la seguridad del trabajo y la vida humana y del programa de ejecución de las medidas que preserven el medio ambiente, son causa de nulidad de los derechos mineros, según lo preceptuado en el Artículo 58, incisos f) e i) de la Ley de Minas.

Lo anterior se complementa con la posibilidad que otorga el sistema contravencional que aplica el Ministerio de Ciencia, Tecnología y Medio Ambiente, que permite a los inspectores estatales de otros Organismos de la Administración Central del Estado, a imponer medidas administrativas por las contravenciones ambientales tipificadas, mediante un convenio previo.

En igual sentido la Resolución No. 223, del Ministerio de Ciencia, Tecnología y Medio Ambiente, Reglamento del control administrativo de fuentes contaminantes generadoras de residuales líquidos y sólidos (2014), persigue como objetivo el control de las fuentes contaminantes que generan residuales líquidos y sólidos que afectan el suelo y las aguas terrestres y marinas, y donde se encuentran precisamente la Industria minera en sus diversas modalidades (a) Explotaciones mineras subterráneas y operaciones conexas y (b) Explotaciones a cielo abierto. Esto es otra muestra más de la protección al medio ambiente, en la actividad minera.

\subsection{Problemáticas reales en la preservación del medio ambiente en la aplicación de la legislación minera}

Hasta aquí hemos analizado el contenido normativo que define cómo se refleja en la legislación minera cubana la protección al medio ambiente, pretendemos a continuación realizar un diagnóstico de la situación actual, partiendo de las principales problemáticas existentes.

Una de las mayores dificultades que en la práctica se enfrenta es lo relacionado con la formación del fondo para la preservación del medio ambiente, ya que el fijar un monto mínimo del 5\% del total de la inversión, teniendo en cuenta que las inversiones en la minería por su naturaleza son millonarias, obliga al concesionario a mantener 
inmóviles grandes sumas de dinero en un momento en que la mayoría de las actividades se sustentan en operaciones crediticias.

De igual manera el país enfrenta otro problema relacionado con las áreas degradadas por actividades mineras realizadas con anterioridad a la promulgación de la Ley de Minas, por entidades ya extinguidas o sin que al presente se pueda identificar su responsable, por lo que es necesario buscar alternativas para su restauración o rehabilitación pudiendo analizarse la posibilidad de utilización del Fondo Nacional del Medio Ambiente, creado mediante la Ley No. 81 Del Medio Ambiente y dirigido a financiar total o parcialmente proyectos o actividades dirigidas a la protección del medio ambiente.

El incumplimiento de lo establecido en los proyectos mineros en cuanto a legislación ambiental ha propiciado, en ocasiones, que los terrenos explotados no sean adecuadamente rehabilitados al cesar las labores de minería (Watson 2008).

Otro aspecto de trascendental importancia a tener en cuenta en la protección al medio ambiente por acciones de la minería, es el auge que ha tenido en los últimos tiempos la extracción de minerales de forma ilegal, sin cumplir con los requerimientos legales establecidos, muestra de ello ha sido la denominada "la fiebre del oro", fundamentalmente en la región oriental del país, lo que ha traído consigo serios daños al medio ambiente, al decir de funcionarios del CITMA y de la Oficina Nacional de Recursos Minerales, provocados fundamentalmente por la falta de conocimientos técnicos para ejecutar una labor que requiere de precauciones extremas.

Aún es insuficiente el control de los poseedores de la tierra, los mismos no ejercen el deber de custodiar estas áreas, alegando en ocasiones no disponer de estructuras específicas para lograr tal fin, lo que no justifica su incumplimiento. Las leyes son precisas en cuanto al deber de proteger el referido patrimonio.

Los efectos de lo que Peña Pupo (2018), jefe de la Unidad de Medio Ambiente del CITMA en Holguín, define como minería asociada a la extracción artesanal de oro, plantean, según dijo, una mirada rigurosa y respuestas severas sistemáticas. Se evidencia 
una práctica destructora la en la extracción ilegal de oro, que afecta de manera directa la calidad del agua, aumentando los sólidos en suspensión en su cauce y además la cantidad removida de desechos será arrastrada inevitablemente con las crecidas del rio incrementando su contaminación. De igual manera de producirse un cambio en las pendientes del terreno puede causar grandes inundaciones, con el consiguiente daño al medio ambiente. Así mismo se han visto afectados algunas especies animales a las que se han modificado su hábitat. Definitivamente para eliminar las secuelas de ese fenómeno se necesita de un plazo prudencial, generalmente largo.

En la práctica existe una voluntad de los concesionarios de cumplir con sus obligaciones ambientales y de las Autoridades Ambiental y Minera, de hacer cumplir las regulaciones encaminadas a la preservación del medio ambiente; de hecho, existen múltiples ejemplos de una oportuna y eficaz rehabilitación tanto en la minería de envergadura como la del níquel, así como en la pequeña minería dirigida a los materiales de construcción.

Pero a pesar de las acciones que se realizan para la protección al medio ambiente, derivado de la minería, se precisa contemplar la aplicación de medidas jurídicas más integrales.

Se debe continuar trabajando en mecanismos y reglamentaciones que permitan una temprana y progresiva rehabilitación paralela a la realización de las actividades mineras, previstas y programadas desde el inicio de la actividad y a partir de los programas directores del territorio, que garanticen además la explotación racional de los recursos minerales y una eficaz gestión ambiental.

De igual manera se requiere de un control preciso para todos aquellos que intervienen en las labores mineras ya sean personas naturales o jurídicas, legales o ilegales.

Es necesario actualizar los instrumentos jurídicos vigentes tales como la Ley de Minas y Su Reglamento y la Ley del Medio ambiente en consonancia con la realidad 
Lafita., $C$., $Y$.

cubana actual y los Lineamientos de la Política económica y social del partido y la Revolución.

\section{5.- CONCLUSIONES}

En Cuba existe una ley marco ambiental, la Ley 81 Del Medio en la que se establecen las principales responsabilidades de los actores económicos en materia ambiental y los derechos y obligaciones de la sociedad en general. Varios de sus artículos se ejecutan por si mismos de los cuales se derivan obligaciones y responsabilidades directas. Se puede considerar que la legislación ambiental cubana es una legislación avanzada, en tanto se ha incorporado el paradigma de la sostenibilidad a nivel constitucional, en la Ley Marco del Ambiente y en la ley de minas.

En Cuba la Ley de Minas cumple dos propósitos, por un lado, promueve y estimula la actividad minera brindando a quien ejerce la actividad minera un régimen jurídico que lo protege y por otro lado garantiza la tutela del medio ambiente.

Considerando que la temática ambiental y minera están en constante desarrollo, los sistemas jurídicos actuales que lo regulan deben irse perfeccionando de manera que se produzcan lo ajustes legislativos necesarios, teniendo en cuenta las experiencias adquiridas mediante la aplicación de un régimen minero ambiental que garantice la seguridad jurídica como consecuencia de la aplicación de las leyes.

\section{REFERENCIAS}

CEPAL. 2013: La sostenibilidad del desarrollo a 20 años de la cumbre para la tierra. Avances, brechas y lineamientos estratégicos para América Latina y el Caribe. [en línea]. Consulta: 11 junio 2018. Disponible en: $\underline{\text { www.cepal.org/rio20. }}$

Constitución de la República de Cuba [Const] de 1976 y 2019.

Decreto Ley No. 222 de 1997. Reglamento de la Ley de Minas. Fecha de promulgación del decreto. Número en el Diario Oficial.

Gómez Tapia, José Luis. (2018). Enfoques epistemológicos de la investigación jurídica. Una aproximación al estado de la cuestión. Revista Ciencia Jurídica y Política. p.p 11-22. 
Recuperado de: $\quad$ https://portalderevistas.upoli.edu.ni/index.php/5 revciencasjuridicasypoliticas/article/view/39

Ley No. 33 de 1981. De Protección del Medio Ambiente y el Uso Racional de los Recursos Minerales. 10 de enero de 1981.

Ley No. 76 de 1994. Ley de Minas. Gaceta Oficial de la República de Cuba, No. 3, La Habana, Cuba, 23 de enero.

Ley No. 81 de 1997 de Medio Ambiente. Gaceta Oficial de la República de Cuba, No. 7, La Habana, Cuba, 11 de julio.

Manzanares Garmendia, Grethy(2020). Desarrollo sostenible y políticas públicas: enfoque de la ONU y ecología política. Revista Ciencia Jurídica y Política. p.p73-87. Recuperado de: $\quad$ https://portalderevistas.upoli.edu.ni/index.php/5revciencasjuridicasypoliticas/article/view/636.

Pavo Acosta, Rolando (2009). La Investigación Científica del Derecho, Fondo Editorial, "Nuevos Tiempos. Nuevas Ideas”, Universidad Inca Gracilazo de la Vega, Lima, Perú.

PEÑA PUPO, Rolber (2018). "La fiebre del oro y sus peligros”. Periódico Granma, Cuba. 2018.. Recuperado de http://www.granma.cu/cuba/2018-03-27/la-fiebre-del-oro-ysus-peligros-27-03-2018-21-03-08 . Revisado 15 abril 2021.

Programa de las Naciones Unidas para el Medio Ambiente (1991). Recuperado en www.pnuma.org

Programa Nacional de Medio Ambiente y Desarrollo. República de Cuba (1993).

Resolución 104 de 2019 [Ministerio de Trabajo y de la Seguridad Social]. Por la cual se establecen las actividades autorizadas para ejercer el trabajo por cuenta propia. 22 de octubre de 2019.

Resolución 158 de 2014[Ministerio de Energía y Minas]. Por la cual se establece el Reglamento de Seguridad Minera. 16 de junio de 2014.

Resolución 11, 2019 [Ministerio de Trabajo y de la Seguridad Social]. Por la cual se establece el Reglamento del ejercicio del trabajo por cuenta propia. 29 de junio de 2018.

Resolución 12, de 2018 [Ministerio de Trabajo y de la Seguridad Social]. Por la cual se establecen las actividades que se pueden ejercer como trabajo por cuenta propia, su 
Lafita., $C$., $Y$.

denominación y alcance. de 29 de junio de 2018.

Resolución 41 de 20132019 [Ministerio de Trabajo y de la Seguridad Social]. Por la cual se establece el Reglamento del ejercicio del trabajo por cuenta propia, 22 de agosto de 2013.

Resolución 42 de 2013 [Ministerio de Trabajo y de la Seguridad Social]. Por la cual se establece las Actividades que se pueden ejercer como trabajo por cuenta propia, su denominación y alcance. 22 de agosto de 2013.

Resolución 32 de 2010 [Ministerio de Trabajo y de la Seguridad Social]. Por la cual se establece el Reglamento para el ejercicio del trabajo por cuenta propia, 7 de octubre de 2010.

Resolución 33 de 2011 [Ministerio de Trabajo y de la Seguridad Social]. Por la cual se establece el Reglamento del Ejercicio del Trabajo por Cuenta Propia. 6 de septiembre de 2011.

Riera, P. et al, (2011). Manual de Economía Ambiental y de los recursos naturales, Ediciones Paraninfo, Madrid.

Rodríguez Gómez, Gregorio et al (2002). Metodología de la investigación cualitativa, Santiago de Cuba, Prograf, Cuba.

Serrano Jiménez, Pablo (1998), Filho Heitor, Pinto, Metodología para las investigaciones jurídicas, Sao Paulo, Brazil.

Serrano Jiménez, Pablo (2015). Metodología para las investigaciones jurídicas, Rio de Janeiro, Brazil.

Unión Mundial para la naturaleza, Programa de las naciones unidas para el medio ambiente y el Fondo mundial para la naturaleza. Cuidar la tierra. Estrategia para el futuro y la vida., (1991).

Villabella Armengol, Carlos M. (2012). La metodología de la investigación y la comunicación jurídica, 2a. edición, Félix Varela, La Habana, Cuba.

WATSON, R (2008). Situación actual y perspectiva de la explotación de yacimientos de materiales de construcción. Inédito, Cuba. 